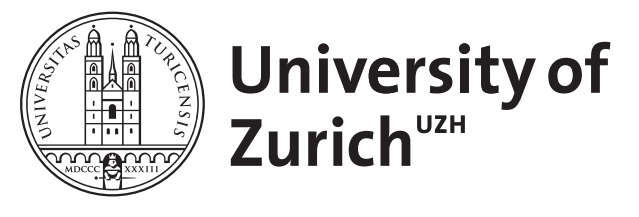

\title{
Retrieving relatives from historical data
}

\author{
Hundt, Marianne ; Denison, David ; Schneider, Gerold
}

\begin{abstract}
Variation and change in relativization strategies has been well documented (e.g. Ball 1996: 46, Biber and Clark 2002, Biber, Johansson, Leech, Conrad and Finegan 1999, Johansson 2006, Lehmann 2002). Certain types of relative clause, namely that-relatives and zero relatives, were difficult to retrieve from plain-text corpora. Studies therefore either relied on manual extraction of data or a subset of possible relativization strategies. In some text types, however, the zero relative is an important member of the class of possible relativizers. Recent advances in syntactic annotation should have made that-relatives and zero relatives more accessible to automatic retrieval. In this article, we test precision and recall of searches on a modest-sized corpus, i.e. scientific texts from ARCHER (A Representative Corpus of Historical English Registers), as a preliminary to future work on the large corpora which are increasingly becoming available. The parser retrieved some false positives and at the same time missed some relevant data. We discuss structural reasons for both kinds of shortcoming as well as the possibilities and limitations of parser adaptation.
\end{abstract}

DOI: https://doi.org/10.1093/llc/fqr049

Posted at the Zurich Open Repository and Archive, University of Zurich ZORA URL: https://doi.org/10.5167/uzh-52961

Journal Article

Accepted Version

Originally published at:

Hundt, Marianne; Denison, David; Schneider, Gerold (2012). Retrieving relatives from historical data. Literary and Linguistic Computing, 27(1):3-16.

DOI: https://doi.org/10.1093/llc/fqr049 


\section{Retrieving relatives from historical data}

2

3 Marianne Hundt, Zürich, David Denison, Manchester and Gerold Schneider, Zürich

4

5 corresponding author:

6 Prof. Dr. Marianne Hundt

$7 \quad$ Englisches Seminar

$8 \quad$ Plattenstrasse 47

9 CH-8032 Zürich

10 m.hundt@es.uzh.ch

11 


\section{Abstract}

13 Variation and change in relativization strategies has been well documented (e.g. Ball 1996: 46, Biber 14 and Clark 2002, Biber, Johansson, Leech, Conrad and Finegan 1999, Johansson 2006, Lehmann 2002, 15 Sigley 1997). Certain types of relative clause, namely that-relatives and zero relatives, were difficult 16 to retrieve from plain-text corpora (Biber 1988, Olofsson 1981). Studies therefore either relied on 17 manual extraction of data or a subset of possible relativization strategies. In some text types, however, 18 the zero relative is an important member of the class of possible relativizers (Ball 1994). Recent 19 advances in syntactic annotation should have made that-relatives and zero relatives more accessible to

20 automatic retrieval. In this paper, we test precision and recall of searches on a modest-sized corpus, 21 i.e. scientific texts from ARCHER (A Representative Corpus of Historical English Registers), as a 22 preliminary to future work on the large corpora which are increasingly becoming available. The 23 parser retrieved some false positives and at the same time missed some relevant data. We discuss 24 structural reasons for both kinds of shortcoming as well as the possibilities and limitations of parser 25 adaptation.

27 Acknowledgement

28 We would like to thank the two anonymous reviewers of Literary and Linguistic Computing for their valuable comments. 


\section{Introduction}

32 Olofsson (1981) is an early corpus-based study that addresses the accessibility of relative clauses to automatic retrieval. His study is based on one of the first standard reference corpora of Present-Day (American) English, the Brown corpus. The corpus was available in a version that had been tagged for parts of speech (POS) but not parsed. POS-tagging, however, is not sufficient for the retrieval of relative clauses. At the same time, relative clauses are too frequent in a corpus of that size (i.e. approximately one million running words) to be extracted manually. Olofsson therefore considered automatic retrieval of the data. He (1981: 14) estimates that $95 \%$ of all occurrences of which are relative pronouns, but less than $20 \%$ of all uses of that are relatives. Even more problematic are zero relatives, as Olofsson (1981: 14) continues to point out:

What deals the final and fatal blow, however, to the idea of letting the computer do the excerption of relative constructions, without pre-editing of the text material, is zero pronoun, which is out of reach as long as syntactic information is not included in the tape fed to the computer.

In the end, he resorted to the manual analysis of a representative sub-corpus, thus somewhat defeating the purpose of a computerized text corpus. Biber (1988), whose aim was to analyse a broad range of features in large corpora, only retrieved overtly marked relative clauses. Ball (1994: 297) discusses zero relatives as one of the problem cases for automatic analysis: "the identification of non-overt elements requires manual effort, a parsed corpus, or a robust parser, but there are considerable accuracy and coverage issues with currently available parsers [...]." She (1994: 301) even concludes that multi-factorial text analyses of the type that Biber (1988) conducted were 'premature' because they excluded zero relatives and thus 
an important part of the possible envelope of variation. An early attempt at using POS-tagged data for present-day English was Lehmann (1997), a more recent one Huber (forthcoming). Lehmann uses 7 tag-based patterns to retrieve NP-NP combinations from a corpus of presentday English (tested against a retrieval strategy that relies on V sequences) and achieves $100 \%$ recall with this. Precision of his retrieval strategy is best for NP-NP strings where the second NP is a pronoun (Lehmann 1997: 185), but it is relatively low at $41 \%$. It can be improved in a corpus that uses different tags for inflected verbs (ibid.: 187). A set of 9 constraints which limit the dataset further (pattern-matching algorithms, using regular expressions) brings recall down to $96 \%$ but precision up to $87 \%$ (ibid.: 191). However, Lehmann points out that the main problem with his study is that recall and precision were tested on the same data set that the retrieval strategy was developed on. Huber (forthcoming) only mentions that he retrieved sequences of two nominals that were manually post-edited but does not report findings on precision and recall for this retrieval procedure.

Recent developments in robust corpus annotation tools have made parsing of corpora much easier. This kind of syntactic annotation, in turn, makes retrieval of zero relatives a more realistic goal. Parser output has only been tested for Present-Day English data, so far. The objective of our paper is to test precision and recall of parser output for relative clauses in parsed historical data. 'Precision' is the technical term used for the targeting potential of a data retrieval procedure. It measures the percentage of true positives in the reported hits, i.e. how many of the automatically reported hits are correct. In other words, it concerns the proportion of 'false positives'. 'Recall' is the term used for the number of relevant strings that the search retrieves, i.e. how many (i.e. per cent) of the relative clauses in the text are found by automatic parsing. Precision usually decreases with an increase in recall (at the cost 
but the focus of this paper is not on parser improvements. We aim foremost to evaluate the usefulness and limitations of automatically retrieving relative clauses from a syntactically annotated corpus.

Our material has been taken from the scientific part of the ARCHER corpus (the forthcoming version 3.2), which we will briefly introduce in section 2 of this paper. We will detail the kinds of relatives we retrieved from the POS-tagged and parsed corpus in section 3. The evaluation of the parser output will present quantitative results on precision and recall as well as a discussion of examples that the parser failed to analyse correctly. We will conclude by making tentative suggestions for future approaches to automatic retrieval of relatives from annotated corpora. ${ }^{1}$

\section{ARCHER and recent developments}

Collaboration and extension of the original ARCHER corpus has been going on for several years. ${ }^{2}$ In our paper we use the science texts of the corpus, including some American English scientific texts for all periods from 1700 onwards that were recently added to the corpus. Table 1 gives an overview of the data.

Table 1: $\quad$ Science texts in ARCHER 3.2 (number of words per sub-period)

\begin{tabular}{|c|c|c|c|c|c|c|}
\hline & $1700-49$ & $1750-99$ & $1800-49$ & $1850-99$ & $1900-49$ & $1950-99$ \\
\hline $\mathrm{AmE}$ & 0 & 20,664 & 20,815 & 21,326 & 20,963 & 25,610 \\
\hline $\mathrm{BrE}$ & 20,780 & 20,565 & 20,994 & 21,715 & 21,337 & 21,308 \\
\hline
\end{tabular}

Our searches were based on a preliminary version of the forthcoming ARCHER 3.2 corpus which includes two additional files for the second half of the twentieth century in the American subpart of the corpus, hence the slightly larger subcorpus in this period. 
97 The science part of the ARCHER corpus was annotated with a probabilistic parser 98 (Pro3Gres) developed by Schneider (2008). The parser uses a dependency-based grammatical 99 model close to Tesnière's Dependency Grammar conception (1959), combining a hand-

100 written competence grammar and probabilistic performance disambiguation learnt from the 101 Penn Treebank (Marcus, Santorini and Marcinkiewicz 1993). It was designed to cover the 102 most frequent phenomena of standard Present-Day English grammar. It is fast (the BNC 103 parses in under a day) and has been evaluated on several genres and varieties (Haverinen, 104 Ginter, Pyysalo and Salakoski 2008, Lehmann and Schneider 2009). It is suitable for parsing 105 different Englishes, as it is robust, so that its output is quite reliable on a number of English 106 varieties (Schneider and Hundt 2009). An evaluation of the performance on subject, object 107 and PP-attachment relations, using the GREVAL gold standard (Carroll, Minnen and Briscoe 108 2003) and 100 random sentences from the BNC, is given in Table 2.

109 Table 2: $\quad$ Performance of the Pro3Gres parser.

\begin{tabular}{|c|c|c|c|c|}
\hline $\begin{array}{l}\text { Performance on GREVAL } \\
\text { (500 sentences) }\end{array}$ & Subject & Object & Noun-PP & Verb-PP \\
\hline Precision & $92 \%$ & $89 \%$ & $74 \%$ & $72 \%$ \\
\hline Recall & $81 \%$ & $84 \%$ & $66 \%$ & $84 \%$ \\
\hline
\end{tabular}

Performance on BNC

(100 sentences)

$\begin{array}{llccc}\text { Precision } & 86 \% & 87 \% & 75 \% & 89 \% \\ \text { Recall } & 83 \% & 88 \% & 77 \% & 70 \%\end{array}$


111 Precision and recall of relative clause parsing were also evaluated for PDE: Schneider (2008:

112188 ) reports $91 \%$ precision and $68 \%$ recall for the anaphora of relative clause subjects (but

113 this only concerns a subset of all possible relative clauses we are interested in here).

\section{Defining the scope of relative clause retrieval}

115 Relativizers in standard PDE include who, whom, whose, which, that, zero, plus relative

116 adverbs where, when, why, whence, ${ }^{3}$ where + Prep (whereby, etc.), plus other possibilities

117 (what, as, etc.) some chiefly dialectal. Most of the first six typically relativize an NP and

118 themselves constitute an $\mathrm{NP}^{4}$ though whose (always) and which (sometimes, though now

119 only rarely) can function as determiners in the relative phrase: ${ }^{5}$

120 (1) In the ${ }_{1}$ paper ${ }_{1}$ whose title is given above the author has shown ... (ARCHER,

$121 \quad$ 1874mall.s6b)

122 (2) there arises ... ${ }_{1}$ the necessity for a new supply of water ..., ${ }_{1}$ which necessity is

123 met by ... (ARCHER, 1886greg.s6a)

124 However, whose and whom are not part of the parser grammar. At the same time, a simple

125 lexical search for these relativizers shows that they are very infrequent in our data, and we

126 therefore feel confident in excluding them from the analysis. ${ }^{6}$

127 Initially, we had planned to retrieve reduced relative clauses as well. These would have

128 been of interest from the methodological angle of this paper because, like zero relatives, they

129 lack an overt relativizer and are thus a challenge for automatic data retrieval. However, they

130 proved to be somewhat problematic from a theoretical point of view, as we will show.

131 Consider these relative clauses, where the relativizer is subject of its clause and is followed

132 by a form of be (examples $3 \mathrm{a}$ and $4 \mathrm{a}$ ); there is a shorter form, more or less synonymous, in

133 which both relativizer and be are missing (examples $3 \mathrm{~b}$ and $4 \mathrm{~b}$ ): 
(3) a. ... those that survived ${ }_{1}$ the shock ${ }_{1}$ which was occasioned by this sudden transition ... (ARCHER, 1874gunt.s6b)

b. ... those that survived the shock occasioned by this sudden transition ...

(4) a. Most of ${ }_{1}$ the studies ${ }_{1}$ which are attempting to move to higher levels of complexity ... (ARCHER, 1975macm.s8b)

b. Most of the studies attempting to move to higher levels of complexity ...

140 Some scholars accordingly describe the latter types, in which a participial phrase 141 postmodifies a noun, as "reduced relatives" (e.g. Quirk, Greenbaum, Leech and Svartvik 1985: 418). Biber et al. explicitly compare them to "full relative clauses" (Biber, Johansson, Leech, Conrad and Finegan 1999: 631-632), which implies that they are reduced relative

144 clauses, though Biber et al. retain the more cautious label "postmodifying participial clauses". Other scholars, e.g. Sag (1997: 433, 471-3), not only use the "reduced relative"

146 label but go further and extend it to postmodifying patterns headed by something other than a participle (examples $5 b$ and $6 b):^{7}$

(5) a. She used an ${ }_{1}$ apparatus ${ }_{1}$ which was similar in principle to ... (ARCHER, 1925dymo.s7b)

(6) a. ... by means of ${ }_{1}$ the visual image, ${ }_{1}$ which was greatly out of focus on account of the ... (ARCHER, 1895keel.s6a) b. ... ?by means of the visual image greatly out of focus...

154 Quirk et al. say of certain types of postmodifying adjective that they "can be seen as reductions of relative clauses" (1985: 1294); examples would be

(7) This fact may probably lead to something useful hereafter. (ARCHER, 1791rush.s4a) 
(8) This stomach possesses a property similar to that of the bladder ... (ARCHER, 1851dadd.s6a)

160 But while some post-nominal PPs can plausibly be regarded as reduced relative clauses (e.g. out of focus in example 6b), others cannot; 15 minutes in (9) cannot be the antecedent of a relative clause:

(9) WE began to look for the first contact of Venus with the Sun, at least 15 minutes (*which were) before the time given by calculation (ARCHER, 1769west.s4a)

Discriminating between those that can and those that cannot is hard, both for humans and parsing programs. It seems, then, that to include putative reduced relative clauses would be to introduce a very fuzzy boundary to our dataset, as well as bringing in numerous examples lacking a relativizer. Huddleston and Pullum refuse to class them as relative clauses "since there is no possibility of them containing a relative phrase" (2002: 1265). But there are further reasons for caution. Quirk et al. observe a couple of properties of postmodifying participial clauses which distinguish them from relative clauses, including participial -ing forms that could not correspond to a progressive verb in a full relative clause (Quirk, Greenbaum, Leech and Svartvik 1985: 1263): 1791rush.s4a)

With non-restrictive postmodification there is the possibility of movement to initial position for the alleged reduced relatives (11b. and 11c.) but not for the full relative clauses (11 d.): (11) a. ... the visual image, 1 which was greatly out of focus, ... (ARCHER, $1895 \mathrm{keel} . \mathrm{s} 6 \mathrm{a})$

b. the visual image, greatly out of focus, ...

c. greatly out of focus, the visual image ... 
d. *which was greatly out of focus, the visual image ...

183 Furthermore, that very mobility "implies that nonfinite non-restrictive clauses are equivocal 184 between adnominal and adverbial role" (Quirk, Greenbaum, Leech and Svartvik 1985: 1271).

185 All in all, then, so-called reduced relative clauses are a rather dubious category. It remains 186 true that postmodifying participial clauses and relative clauses can often be seen as linguistic 187 alternatives. Because of the theoretical and practical problems outlined above, however, we 188 decided to narrow our definition of the variable somewhat and exclude reduced relative 189 clauses from our study.

190 Our study is part of a larger project on developments in noun phrase complexity in the 191 late Modern period (Hundt, Denison and Schneider in prep.). We therefore confine attention 192 to adnominal relative clauses, i.e. those with an NP as antecedent. ${ }^{8}$ By focusing on adnominal 193 relative clauses, we are looking at data sets that are comparable with previous studies and 194 avoid unwanted statistical noise (see Sigley 1997: 37-40). The adnominal relative clauses we 195 include in our study are introduced either by a standard ${ }^{9}$ English wh-pronoun (i.e. who and 196 which) or that; in addition, we also retrieved zero relatives automatically.

\section{Evaluation of parsed data}

198 We initially analysed for precision some concordances (AmE data from the 1700s) that were 199 retrieved from the parsed corpora. After the parser had been adapted, we tested precision on a 200 larger set of concordances across both BrE and AmE as well as across time. Note that we 201 only tested for precision of relative clause parsing, not for precision of completely parsed sentences. The VP in the following example, for instance, was analysed wrongly in our output files with be as the head of the VP rather than perforate, but the relative clause was correctly identified: 
(12) The effects of a yearly discharge of sap from the tree in improving and increasing the sap is demonstrated from the superior excellence of those ${ }_{1}$ trees which have been perforated in an hundred places, by a small wood-pecker which

Ball (1994: 295) claims that recall is more difficult to assess in large corpora than precision:

210 "it is generally impossible for the analyst to know what has been missed without analysing

211 the entire corpus by hand." Such an extreme view tacitly assumes that it is impossible to extrapolate recall tested on a small set of texts to the corpus as a whole. While an exact measure of recall is indeed impossible to attain, and it is impossible anyway for other reasons

214 like inter-annotator agreement, we would like to claim that testing recall in a subset of the 215 corpus analysed automatically can give a good indication of recall for the corpus as a whole.

216 The recall evaluation on the subset is as reliable as the subset is representative of the whole 217 corpus. In addition to testing for recall of all adnominal relative clauses by analysing a subset 218 of data we have also tested recall for the most frequent relativizer in scientific writing, 219 namely which. As a first step we thus manually annotated all relatives (who, which, that and 220 zero) in a subset of files and verified whether these had been identified by the parser by cross-checking the manually retrieved relative clauses with those listed in the concordances. The subset consisted of a total of 13 files in all, five texts from the 1700s (approx. 10,000 words) and 4 texts each for the 1800 s and 1900s (i.e. approx. 16,000 words for the two subperiods together). As with precision, we only tested for recall of relative clauses, not for correctly parsed sentences. The antecedent in the following example was given as what (which could, arguably, be correct), but a much more obvious antecedent for the relative clause would be $i c e:^{10}$ 
(13) The ice-makers attended the pits usually before the Sun was above the horizon, and collected in baskets what was frozen, by pouring the whole contents of the pans into them and thereby retaining the ${ }_{1}$ ice, ${ }_{1}$ which was daily conveyed to the grand receptacle or place of preservation [...] (ARCHER, 1775bark.s4b).

In another instance, the parser had wrongly picked out a relativizer (who) as the antecedent of another relativizer (which):

(14) A particularly favourable one has been afforded me lately through the kindness of ${ }_{1} \mathrm{Mr}$. GORDON, ${ }_{1}$ who has furnished me with considerable quantities of a ${ }_{2}$ fluid obtained during the compression of oil gas, 2 of which I had some years since possessed small portions, sufficient to excite great interest, but not to satisfy it. (ARCHER, 1825weav.s5b)

This was counted as a relevant example despite the fact that antecedent had not been correctly identified. ${ }^{11}$ There are also examples where the verb of the relative clause was not identified correctly, and it would thus (in theory) be impossible to decide which relative clause was correctly parsed, as in the following example (where the parser misanalysed the inflectional ending ' $d$ as a reduced form of the verb have because it is part of the lexicon implemented in the parser): ${ }^{12}$

(15) An irregular gust of Wind blowing upon and shaking the Columns, was (I suppose) the Cause of that ${ }_{1}$ trembling, ${ }_{1}$ which appear'd in the triangular Streams, and the ${ }_{2}$ Cause also ${ }_{2}$ which destroy'd that fine appearance of the Canopy. (1720cote.s3b)

In this particular instance, two relative clauses with relativizer (which) and verb ('d) were retrieved from the corpus. In other words, the example did not prove a problem for the evaluation of recall. 
In the following, we will first present the overall results on precision and recall and then

253 turn to the discussion of individual examples.

\subsection{Quantitative results}

Overall, the initial results for precision were $83.5 \%$ for relatives introduced by who/which/that and $18.5 \%$ for zero relatives; recall was $43 \%$. The parser was subsequently adapted with the help of the comments in the concordances.

The parser grammar was adapted in several ways, some directly addressing shortcomings in the analysis of relative clauses, some generally improving performance, and some addressing phenomena that are more frequent in previous stages of English. Perhaps surprisingly, none of the changes addresses features that no longer exist in PDE. Each change turned out to be a general parser improvement. The parser was adapted in the following five ways:

264 First, whom and whose were added as relativizers, correcting a shortcoming of the parser 265 that is relevant for earlier stages of English in general (even though they occurred 266 infrequently in our scientific data).

267 Second, the parser grammar was adjusted so that pied-piping constructions are now parsed correctly. Previously, relative pronouns inside a prepositional phrase were explicitly disallowed to post-modify NPs, as is the case in pied-piping constructions. This poses problems for sentences such as the following example:

(16) He informed me that in his journey from Passy to Havre de Grace, last summer, he found the ${ }_{1}$ country ${ }_{1}$ through which he travelled, unusually sickly with fevers. (ARCHER, 1786rush.s4a) 
274 In the parser output before adjustment, the relative pronoun which directly attached to

275 country instead of the preposition through. Pied-piping constructions are more frequent in

276 historical data. This adaptation generally improves the parser output. For ARCHER, it was

277 the single most beneficial adaptation made.

278 Third, candidates for relative pronouns acting as objects are only allowed to attach if no overt

279 object exists. In the following sentence, the original parse had the verb represent taking two

280 objects, namely mountains and the relative pronoun object that.

281 (17) He gives the preference to the Gregorian, and mentions as a principal defect of the

282 Cassegrain telescope, that it represents the mountains in the moon as vallies, and

283 the contrary. (ARCHER, 1786ritt.s4a)

284 Valency checks in principle forbid several objects, but long-range objects such as relative

285 pronouns were not sufficiently subjected to these checks. After the correction, this example is

286 no longer incorrectly reported as a relative clause, but correctly as a subordinate clause. This

287 adaptation, in addition to improving performance on historical English, also minimally

288 improves parser performance on PDE.

289 Fourth, a list has been created of words that are licensed to be complementizers. In the

290 Penn Treebank tagset, which the parser uses, the tag IN is used for complementizers,

291 prepositions and relativizers. In rare cases, relative pronouns like which were analysed as

292 complementizers. However, the ambiguity between complementizer and relative pronoun,

293 particularly of that, is still a major source of errors, which brings us to our next point.

294 Fifth, the class of nouns that can introduce subordinate clauses is now learnt from the

295 Penn Treebank, while previously a small, closed list was used. This adaptation only leads to

296 minimal improvements, as the ambiguity depends on semantics and verb valency in ways that

297 the parser does not sufficiently respect. In The suggestion that we should follow we probably 
298 have a relative clause, while in The suggestion that we should go we probably have a

299 subordinated clause. In real world sentences, which are usually more complex than invented 300 examples, attachment ambiguities and the complementizer/relative pronoun ambiguity

301 combine forces. In the following example, the parser attached that to part as relativizer

302 instead of attaching it to consequence as a complementizer.

303 (18) It is in consequence of the sap of these trees being equally diffused through every

304 part of them, that they live three years after they are girdled [...]. (ARCHER, $305 \quad$ 1791rush.s4a)

306 In the next example, the parser attached that to tube as relativizer instead of to found as 307 complementizer:

308 (19) And accordingly I found, upon taking out both of the glasses, and looking through

309 the open tube, that the hearth appeared as perfectly, and as constantly in its

310 unnatural state by reflected light [...]. (ARCHER, 1786ritt.s4a)

311 Parser adaptation led to improved precision ( $85 \%$ for $w h$-/that relatives and $28 \%$ for zero

312 relatives) and recall (53\%) for the 1700s American English data. Table 3 below gives a more

313 detailed summary of the results for the two varieties and across time.

314 Table 3: $\quad$ Precision (after parser adjustment) ${ }^{13}$

\begin{tabular}{|l|l|l|l|}
\hline AmE & $1700 \mathrm{~s}$ & $1800 \mathrm{~s}$ & $1900 \mathrm{~s}$ \\
\hline wh-/that & $85 \%$ & $83 \%$ & $88 \%$ \\
\hline zero & $28 \%$ & $11 \%$ & $11 \%$ \\
\hline BrE & & & \\
\hline wh-/that & $86 \%$ & $82 \%$ & $82 \%$ \\
\hline zero & $20 \%$ & $24 \%$ & $0 \%$ \\
\hline \hline
\end{tabular}




\begin{tabular}{|l|l|l|l|}
\hline \hline pooled results (AmE and $\mathrm{BrE})$ & & & \\
\hline wh-/that & $86 \%$ & $83 \%$ & $86 \%$ \\
\hline zero & $23 \%$ & $18 \%$ & $5 \%$ \\
\hline
\end{tabular}

316 As expected, the parser performs best on overtly marked relative clauses. Note that the parser

317 was adjusted on the basis of the 1700 s data and improvement led to better performance in this

318 period, also for zero relatives. Precision for overtly marked relative clauses is also quite high

319 for the 1800 s and 1900 s, but for zero relatives the figures remain quite low. We doubt,

320 however, whether parser adjustment to data retrieved for the latter two sub-periods would

321 substantially improve the precision of zero-relative retrieval.

322 Recall is much lower, overall, than precision for overtly marked relative clauses, and

323 it is better for the twentieth-century data than for the earlier periods, as Table 4 shows:

324 Table 4: $\quad$ Recall (after parser adjustment; both varieties)

325

\begin{tabular}{|l|l|l|}
\hline sub-period & correctly identified relatives & recall \\
\hline $1700 \mathrm{~s}$ & 40 out of 92 & $43 \%$ \\
\hline $1800 \mathrm{~s}$ & 29 out of 71 & $41 \%$ \\
\hline $1900 \mathrm{~s}$ & 40 out of 76 & $53 \%$ \\
\hline
\end{tabular}

326 Breaking down recall errors by relative pronoun reveals that, quantitatively, the impact of

327 missed zero or that relatives is small. In fact, the majority of relatives that were not retrieved

328 automatically are $w h$-relatives (see Table 5), the most commonly used relativizer in the

329 science part of ARCHER overall:

330 Table 5: $\quad$ Missed relative clauses by relativizer 


\begin{tabular}{|l|c|c|c|l|}
\hline sub-period & that & which/who & zero & total \\
\hline 1700 s & 4 & 46 & 2 & 52 \\
\hline 1800 s & 2 & 39 & 1 & 42 \\
\hline 1900 s & 9 & 25 & 0 & 36 \\
\hline
\end{tabular}

331 In absolute terms, then, the relative clauses that are particularly difficult to retrieve

332 automatically (i.e. that- and zero-relatives) turn out to perform quite well in science texts

333 with respect to recall. In absolute terms, the easy-to-retrieve wh-relativizers are missed more

334 often than the ones that are difficult to retrieve automatically. As recall of relativizer which

335 seemed especially low (probably also because which is a particularly frequent relativizer in

336 our data), we decided to retrieve all instances of which from the bare-text version of our

337 corpus, manually delete all instances that were not adnominal relative clauses, in order to test

338 more widely for recall of this important relativization strategy in historical scientific writing.

339 The results are given in Table 6.

340 Table 6: $\quad$ Recall for adnominal relatives introduced by which

$(\mathrm{a}=\text { automatically retrieved, } \mathrm{m}=\text { manually retrieved })^{14}$

\begin{tabular}{|l|l|l|l|l|l|l|l|l|l|}
\hline & \multicolumn{3}{|l|}{$1700 \mathrm{~s}$} & \multicolumn{4}{l|}{$1800 \mathrm{~s}$} & \multicolumn{2}{l|}{$1900 \mathrm{~s}$} \\
\cline { 2 - 10 } & $\mathrm{a}$ & $\mathrm{m}$ & recall & $\mathrm{a}$ & $\mathrm{m}$ & recall & $\mathrm{a}$ & $\mathrm{m}$ & recall \\
\hline AmE & 110 & 181 & $61 \%$ & 160 & 367 & $44 \%$ & 127 & 224 & $57 \%$ \\
\hline BrE (50s-99s) & 103 & 174 & $59 \%$ & 87 & 180 & $48 \%$ & 59 & 111 & $53 \%$ \\
\hline
\end{tabular}

343 Even though relative clauses introduced by which make up a large part of the missed relative

344 clauses numerically, recall for these relative clauses in a larger section of the corpus is above

345 that for all types of relative clauses considered in our paper. 
A closer look at individual examples from the concordances for both precision and recall

will show where some of the potential problems for parsing lie.

\subsection{Discussion: Precision}

349 One of the reasons why precision with zero relatives is so low in the historical data is that

350 fronting of objects is still common in earlier texts. Examples (20)-(24) are typical instances of

351 false positives (false antecedents are given in italics, erroneously retrieved relative clauses are underlined):

(24) The Places of these two Stars I have not yet observed. (ARCHER, 1724brad.s3b)

364 The parser identifies these fronted objects as antecedents of a zero relative. This is a further

365 task for future parser adaptation. However, the vast majority of false positives defy neat 366 classification. The following is just one example of a real problem case:

(25) The present research forms part of a wider investigation of terrestrial 

atmosphere, and with the systematic motions of the upper atmosphere. (ARCHER, 1925chal.s7b)

372 The parser identified object as the antecedent of the relative clause, associate as its verb and

373 which as the relativizer, but of course the verb is is and the antecedent investigation, in a

374 structure which the Huddleston, Pullum \& Peterson (2002: 1040-1042) would describe as 375 involving (twofold) upward percolation.

\subsection{Recall}

377 As far as recall is concerned, sentence length and complexity in early scientific writing pose 378 obvious problems. Ambiguity increases exponentially when sentences are long. Example (26)

379 illustrates the problem of sentence length; the sentence contains three adnominal relative 380 clauses, two of which were retrieved automatically. (We enclose undetected relative clauses 381 in square brackets):

382 (26) The ${ }_{1}$ opinion, therefore, ${ }_{1}$ which I have formed from what I have hitherto seen is, 383 that the boiled and common water differ from one another in this respect; that 384 whereas the common water, when exposed in a state of tranquillity to ${ }_{2}$ air ${ }_{2}$ that is 385 a few degrees colder than the freezing point may easily be cooled to the degree of such air, and still continue perfectly fluid, provided it still remain undisturbed; the boiled water, on the contrary, can not be preserved fluid in these circumstances; but when cooled down to the freezing point, if we attempt to make it in the least colder, a part of it is immediately changed into ice; after which, by the continued action of the cold air upon it, more ice is formed in it every moment, until the whole of it be gradually congealed before it can become 
393 Sentence complexity is generally a problem because it inflates ambiguity. In principle, the parser can cope with any depth of nesting or stacking, but because this increases syntactic ambiguity, nesting and stacking also pose problems for parsing. In a configuration where two relative clauses follow a noun phrase, for instance, the second relative clause can potentially modify any noun phrase in the first relative clause or even the initial NP. Thus, in the following example the first relative clause (introduced by that) was identified correctly by the parser, but the second one - separated by a series of other subordinate clauses - was not:

(27) The first ${ }_{1}$ rudiments of this art ${ }_{1 \mathrm{a}}$ that I acquired was from the two Hunters, known through all Europe for their superior skill in anatomy, and acting as practical dissector to the celebrated doctors Colignon and Smith, professors of anatomy in

The bracketed that-relative in the following example is nested within another relative clause and might have been missed by the parser for that reason:

(28) several other ${ }_{1}$ Striae were discharged from behind the dark Basis, iwhich intersecting with ${ }_{2}$ others, ${ }_{2}[$ that at the same time arose about the East and West Points,]form'd in the Zenith, or rather 6 or 8 degrees to the South thereof, a second much more elegant and surprizing than the former, and indeed than ${ }_{3}$ any thing

413 The following is a particularly complex example with four relative clauses, of which one (in 414 brackets) was not identified by the parser:

415 (29) The process results in the production of a ${ }_{1}$ form ${ }_{1 \mathrm{a}}$ which I proposed to call the 
Planula, but $1 \mathrm{~b}[$ which Professor HAECKEL has better termed the Gastrula], reserving the former name for a ${ }_{2}$ condition of the Gastrula ${ }_{2 a}$ which sometimes presents itself ${ }_{2 \mathrm{~b}}$ in which there is no aperture of invagination. (ARCHER, 1874lank.s6b)

Particularly difficult to evaluate were cases in which an antecedent was followed by two relative clauses that were introduced by the same relativizer each time. The probabilistic model of the parser includes distance (measured in chunks) which often (but not always) means that close attachments are preferred over more distant ones. The following example occurred in our list of automatically retrieved relative clauses only once with the verb be as the verb of the relative clause; in other words, the second relative clause, introduced by through which, was not parsed correctly (and thus not retrieved):

(30) That all those ${ }_{1}$ parts of any animal Body, ${ }_{1 \mathrm{a}}$ which are vascular, or ${ }_{1 \mathrm{~b}}[$ through which any Fluid passeth,] from the intestines to the minutest Fibre, are the seat of medicine's Operation. (ARCHER, 1720cote.s3b)

At other times, distance from the antecedent did not cause a problem for the parser. The following example was parsed correctly, for instance:

(31) As to the Knife, it was not the Blade, but the ${ }_{1} \mathrm{Haft}$, and the ${ }_{2} \mathrm{Hinge}{ }_{2}$ that goes into it, ${ }_{1}$ which was found shiver'd in Pieces. (ARCHER, 1725nett.s3b)

Note that in example (29) above, the relative clause headed by a preposition (subscript 2b) was parsed correctly. The missed relative clauses in both (29) and (30) are preceded by a coordinating conjunction. Co-ordination creates serious problems for the parser, since the conjunction can combine elements at any level (NP, VP, clause). Statistical models are not of much help here, as there is typically no lexico-grammatical preference. A third example where the second relative clause was missed seems to confirm this: 
(32) A young Scotch ${ }_{1}$ fir, ${ }_{1 \mathrm{a}}$ which had two compleat shoots and a third growing, and $1 \mathrm{~b}$ [which consequently was in its third year], was put into the cold ${ }_{2}$ mixture ${ }_{2}$ which was between 15 and 17. (ARCHER, 1775hunt.s4b)

443 But missing coordinating conjunctions between double relative clauses, likewise, pose a 444 problem for automatic retrieval of relative clauses; in the following example, only the first 445 but not the second relative clause (in brackets) was retrieved in our parser-based approach:

(33) The kind of ${ }_{1}$ preparations of those ${ }_{2}$ parts of the animal body ${ }_{2}[$ which admit of it] ${ }_{1}$ that I now propose to explain, namely by injection and corrosion, exceeds in beauty, nicety and usefulness, that which is commonly called dissection. (ARCHER, 1786morg.s4a.txt)

Rissanen (1984: 424, exx 4 and 5) points out that relative clauses which are close to their antecedent are more likely to be introduced by zero or that and that, with growing distance between antecedent and relativizer, the likelihood increases that the semantically more transparent $w$ h-relativizers will be used. ${ }^{15}$ This is illustrated by the following example of a double relative clause:

(34) Two left off feeding; these I placed on the ${ }_{1}$ racks ${ }_{1 \mathrm{a}} \varnothing$ I had made, ${ }_{1 \mathrm{~b}}$ which I fixed in glass bottles to prevent the worms from getting off: [...] (ARCHER, 1769bart.s4a)

457 But we also came across an interesting counter-example to the general principle that closeness to the antecedent favours zero and that-relatives and that with growing distance, the more explicit wh-relatives are preferred. In example (33) above, the hierarchically second

460 relative clause is introduced by a $w h$-relativizer but the hierarchically first relative clause (i.e. 461 the one with the antecedent the kind of preparations) actually follows it and is introduced by 462 the less explicit that. In other words, a more semantically transparent relative construction is 
463 nested within a long-distance head-relativizer sequence which, surprisingly, has that as 464 relative pronoun.

465 Punctuation in relative clauses in previous stages of the language was somewhat different 466 from the conventions in PDE (see Denison and Hundt in prep.). The prescriptive rule of 467 separating only non-restrictive relative clauses by a comma was not firmly in place yet. Even 468 zero relative clauses, which are always restrictive, are sometimes set off from the remaining 469 text by commas, and therefore the parser grammar has missed the following relevant instance 470 (it only has a rule for zero relatives without a comma):

(35) The first ${ }_{1}$ Experiment, ${ }_{1}[\varnothing$ I have to offer to your Observation at present, $]$ is made on the New England Cedar, or rather Juniper, grafted on the Virginia; and what is remarkable in it, is, That the ${ }_{2}$ Branch, ${ }_{2}$ which is grafted, is left several Inches below the Grafting, which part continues growing as well as the upper Part above problems for the parser. Retrieving relatives that are obsolete (or obsolescent) in PDE would 478 be a case in point. In the following example, the antecedent calculations is repeated after the relativizer:

(36) In the report of March, 1812, page 9, the commissioners gave ${ }_{1}$ calculations on the expense of conveyance of canals, ${ }_{1}$ which calculations were drawn from the experience acquired on canals in England, as to the quantity of work that two

484 A similar 20th-century example does not have a repeated antecedent, and indeed it is not 485 clear whether the relative is adnominal at all: 
(37) In 1849, Carpenter began the study of the wall structure of Foraminifera, restricted largely to the large, calcareous forms, which work was completed in his important "Introduction to the Study of Foraminifera”, in 1862. (ARCHER, 1928gall.s7a)

Adding a repetition rule to the parser grammar for earlier historical texts might even have a negative effect on parser performance.

The following example is no longer possible in PDE because the antecedent would have to

be nominal and the relativizer would have to be preceded by a preposition (i.e. the promotion of which): $:^{16}$

(38) The manufacturing interest, to promote which is one of the objects of the society, is a subject of much importance, and would furnish matter far beyond the limits of an address. (ARCHER, 1823adam.s5a)

Finally, punctuation might again account for the fact that the following restrictive relative headed by which was not retrieved by the parser:

(39) I was at Gibraltar when this happen'd, where I saw above 100 of the Butts of that ${ }_{1}$ Cargo of Brandy, 1 [which were sent thither from Tangier $]$; I likewise spoke with the ${ }_{2}$ Captain of the Dutch Ship, ${ }_{2}$ who told the Governor, myself, and many others where his Vessel sunk; and her rising afterwards at Tangier, appear'd very unaccountable to us, as it does to me to this Day; for there's no Doubt but the Ship

In this example, the correct attachment could have been found by the grammar, but apparently it was judged to be less likely.

508 Sentential relatives are also a problem for automatic retrieval: the distinction between 
510 syntactic, and the parser therefore always attaches relative pronouns to a noun, which means

511 that all sentential relatives get an incorrect parser analysis and have to be removed manually

512 from the parser output. In the following example, which is attached to glass instead of to the

513 clause introduced by from. As it is a relatively long sentence, which is typical for the period,

514 attachment ambiguities multiply.

515 (40) When I expected the flies were near coming out, I tacked coarse cloths up against

516 the windows on the inside, not only to darken the room, but also for the flies to

517 settle on, and to prevent them, in attempting to make their escape, from 1 beating

518 their legs and wings to pieces against the glass, ${ }_{1}$ which I found to be the case last

$519 y e a r$, and which it is probable, prevented their copulating. (ARCHER,

$520 \quad 1769$ bart.s4a)

521 Another problem for the parser is the expression so ... that: it is not included in the parser,

522 partly because few multi-word expressions are recognised, and partly because PDE newspaper and science texts, on which the parser was developed, do not contain many so ...

524 that constructions. In ARCHER, however, they frequently cause parser errors. In the 525 following example, that gets attached to matter.

526 (41) My answer gave him so much satisfaction in the matter, that he immediately sent

527 his orders to his correspondent in London, to procure the instruments. (ARCHER, $528 \quad 1769$ west.s4a)

529 The parser treated semi-colons as a sentence boundary, yet relative clauses in early texts are 530 occasionally punctuated off by a semi-colon, as in the following example:

531 (42) between N.W. by North, and W.N.West, we found the Representation of a very bright Crepusculum, such as ${ }_{1}$ that which appears about 20 Minutes after Sun-set; 
535 This detail had not been changed in the first adaptation of the parser. Future parser

536 adaptation in this direction (also allowing for relative clauses to be separated from the

537 previous clause by a colon or to occur in parentheses) is expected to improve recall of

538 non-restrictive $w h$-relative clauses, i.e. those that tended to be set off by other punctuation

539 than a comma in our earlier texts on a preliminary search.

\section{Conclusion}

541 The aim of our paper was to evaluate the possibilities and limitations of retrieving relative

542 clauses from a parsed corpus of historical English. We found that, initially, precision was

$54383.5 \%$ for overtly marked relatives and but as low as $18.5 \%$ for zero relatives; recall was

$54443 \%$. Parser adjustment improved precision for overtly marked relatives somewhat. For zero

545 relatives, the precision could be improved to $28 \%$ for American scientific texts of the $1700 \mathrm{~s}$,

546 but was found to be as low as $0 \%$ for the British texts of the $1900 \mathrm{~s}$. We found that the parser

547 identified fronted objects as antecedents of zero relatives. Future parser adaptation is

548 therefore likely to slightly improve the accuracy of the parser output in this area and thus

549 improve precision. The main problem will be that the majority of false positives of zero

550 relative clauses defy easy classification and thus do not substantially contribute to parser

551 improvement. Despite adaptations, many seemingly simple problems with parsing historical

552 data persist, such as the complementizer ambiguity (see the discussion of examples 18 and 19

553 above), which poses a problem for precision. With respect to recall, the qualitative analysis of

554 the parser output showed that sentence length and complexity (including nesting) were the

555 most obvious problems for parser failure. 
556 One of the potential advantages of working with parsed data is that it allows one to 557 retrieve zero relatives. These relative clauses remain problematic because precision for zero 558 relatives is so low. Recall for zero relatives in our scientific data, on the other hand, was quite 559 good, but this was mainly because zero relatives are infrequent in this kind of data. They are 560 thus more likely to be a problem for more informal written text types and spoken data and 561 future work on parser adaptation should therefore include these text types. A combination of 562 the tag-based approach described in Lehmann (1997) with the parser-based approach taken in 563 this paper might improve both precision and recall of automatically retrieved zero relatives. 
${ }^{1}$ More details on adapting the parser to historical texts are given in Schneider (2011).

${ }^{2}$ For the development of the ARCHER corpus, see Yáñez-Bouza (2011).

${ }^{3}$ Huddleston, Pullum and Peterson (2002: 1051) include while in this list.

${ }^{4}$ Note that the NP belongs to a PP in the case of pied piping (an instance of 'upward percolation' in the terminology of Huddleston, Pullum and Peterson (2002: 1040)).

${ }^{5}$ Relative clauses and their antecedents are marked by subscripts throughout this paper.

${ }^{6}$ On the choice of who vs. whom, see for instance Tieken-Boon van Ostade (1990) or Schneider (1992a, 1992b).

${ }^{7}$ In fact in early transformational grammar there was a move to derive all attributive adjectives, even premodifying ones, from predicative adjectives in relative clauses (e.g. ... an apparatus which is/was similar $\Rightarrow$ an apparatus similar $\Rightarrow$ a similar apparatus) (see e.g. Culicover 1982).

${ }^{8}$ Sentential relative clauses (e.g. He manages to swim the whole length of the pool, which amazes me) and free relatives (e.g. We will be working on this on Saturday, which will be nice or He always gets what he wants) are examples of relative clauses that are not adnominal. They are likely to have undergone significant changes in the late Modern English period, too, but this will have to be the topic of future research.

${ }^{9}$ It is highly unlikely that non-standard relativizers like what or as would be used in scientific English.

${ }^{10}$ Incorrectly identified antecedents are highlighted in bold throughout the paper.

${ }^{11}$ Note that the parser failed to identify the relative clause with Mr. Gordon as antecedent! 
${ }^{12}$ As part of further improvement in the annotation of ARCHER, spelling variation in the corpus was normalized with VARD (see Schneider 2011). Running the parser over PDE spelling variants significantly improves the overall performance of the parser.

${ }^{13}$ For a table with frequencies of positives and false positives, see the appendix.

${ }^{14}$ Note that the figures for the BrE part of the corpus are based on the data from the second half of the century only.

${ }^{15}$ See also Quirk (1957: 106 n.8) on educated spoken English: "The preponderance of wh- is at its greatest in the cases of double restriction (as in 'there are certain activities which are not scientific which are very important to the human race'), where the second clause has wh- 15 times as against that 5 times and no examples of zero."

${ }^{16}$ An alternative pattern using promote as a verb would be The manufacturing interest, which it is one of the objects of society to promote... 


\section{References}

Ball, C.N. (1994) 'Automated text analysis: Cautionary tales'. Literary and Linguistic Computing 9(4): 295-302.

Ball, C.N. (1996) 'A diachronic study of relative markers in spoken and written English'. Language Variation and Change 8(227-258.

Biber, D. (1988) Variation across speech and writing. (Cambridge, etc: Cambridge University Press).

Biber, D. and Clark, V. (2002) 'Historical shifts in modification patterns with complex noun phrase structures' in T. Fanego et al. (eds.), Historical shifts in modification patterns with complex noun phrase structures. Amsterdam and Philadelphia PA: John Benjamins, pp. 43-66.

Biber, D., Johansson, S., Leech, G., Conrad, S. and Finegan, E. (1999) Longman grammar of spoken and written English. London: Longman.

Carroll, J., Minnen, G. and Briscoe, E. (2003) 'Parser evaluation: Using a grammatical relation annotation scheme' in A. Abeillé (ed.), Parser evaluation: Using a grammatical relation annotation scheme. Dordrecht: Kluwer, pp. 299-316.

Culicover, P.W. (1982) Syntax (2nd Edition). (New York: Academic Press).

Denison, D. and Hundt, M. (in prep.) 'Defining relatives'.

Haverinen, K., Ginter, F., Pyysalo, S., Salakoski, T. (2008) 'Accurate conversion of dependency parses: targeting the Stanford scheme.' Proceedings of third international symposium on semantic mining in biomedicine (SMBM 2008), Turku, Finland, pp. 133-136. 
589 Huddleston, R. (2002) 'Non-finite and verbless clauses' in R. Huddleston \& G.K. Pullum (eds.), Non-finite and verbless clauses. Cambridge: Cambridge University Press, pp. $1171-1271$.

Huddleston, R., Pullum, G.K. and Peterson, P. (2002) 'Relative constructions and unbounded dependencies' in R. Huddleston \& G.K. Pullum (eds.), Relative constructions and unbounded dependencies. Cambridge: Cambridge University Press, pp. 1031-1096.

Hundt, M., Denison, D. and Schneider, G. (in prep.) 'Relative complexity in scientific discourse'. English Language and Linguistics

Johansson, C. (2006) 'Relativizers in nineteenth-century English' in M. Kyto et al. (eds.), Relativizers in nineteenth-century English. Cambridge: Cambridge University Press, pp. 136-182.

Lehmann, H.M. (1997) 'Retrieval of zero elements in a computerised corpus' in M. Ljung (ed.), Retrieval of zero elements in a computerised corpus. Amsterdam: Rodopi, pp. 179-194.

Lehmann, H.M. (2002) 'Zero subject relative constructions in American and British English' in P. Peters et al. (eds.), Zero subject relative constructions in American and British English. Amsterdam and New York: Rodopi, pp. 163-177.

608

Lehmann, H.M. and G. Schneider. (2009) 'Parser-based analysis of syntax-lexis interaction' in A.H. Jucker et al. (eds.), Parser-based analysis of syntax-lexis interaction. Amsterdam and New York: Rodopi, pp. 477-502.

Marcus, M.P., Santorini, B. and Marcinkiewicz, M.A. (1993) 'Building a large annotated corpus of English: The Penn Treebank'. Computational Linguistics 19(2): 313-330. 
612 Olofsson, A. (1981) Relative junctions in written American English. (Gothenburg: Acta

613 Universitatis Gothoburgensis).

614 Quirk, R. (1957) 'Relative clauses in educated spoken English'. English Studies 38(97-109.

615 Quirk, R., Greenbaum, S., Leech, G. and Svartvik, J. (1985) A comprehensive grammar

616 of the English language. (London and New York: Longman).

617 Rissanen, M. (1984) 'The choice of relative pronouns in 17th century American English' in J.

618 Fisiak (ed.), The choice of relative pronouns in 17th century American English. Paris

619 and The Hague: Mouton, pp. 417-435.

620

Sag, I.A. (1997) 'English relative clause constructions'. Journal of Linguistics 33(431-483.

621

622

623

624

625

626

627

628

629

630

631

632

633

634

635

Schneider, E.W. (1992a) 'Who(m)? Case marking of wh-pronouns in written British and American English' in G. Leitner (ed.), Who(m)? Case marking of wh-pronouns in written British and American English. Berlin and New York: Mouton de Gruyter, pp. 231-245.

Schneider, E.W. (1992b) 'Who(m)? Constraints on the loss of case marking of wh-pronouns in the English of Shakespeare and other poets of the Early Modern English period' in M. Rissanen et al. (eds.), Who(m)? Constraints on the loss of case marking of whpronouns in the English of Shakespeare and other poets of the Early Modern English period. Berlin and New York: Mouton de Gruyter, pp. 437-452.

Schneider, G. (2008) 'Hybrid long-distance functional dependency parsing'. PhD, University of Zürich.

Schneider, G. (2011) 'Adapting a parser to historical English'. Paper presented at the Helsinki Corpus Festival, September 27 - October 2, 2011.

Schneider, G. and M. Hundt. (2009) 'Using a parser as a heuristic tool for the description of New Englishes'. Paper presented at CL 2009, Liverpool. 
636 Sigley, R. (1997) 'Choosing your relatives: Relative clauses in New Zealand English'. PhD, 637 Victoria University.

638 Tesnière, L. (1959) Eléments de syntaxe structurale. (Paris: Librairie Klincksieck).

639 Tieken-Boon van Ostade, I. (1990) 'Betsy Sheridan: Fettered by grammatical rules?'.

$640 \quad$ Leuvense Bijdragen 79(79-90.

641 Yáñez Bouza, N. (2011) 'ARCHER past and present (1990-2010)'. ICAME Journal 35(205$642 \quad 236$.

643

644

645 
646 Appendix

647 Table: Frequencies of relative clauses after parser-adjustment

\begin{tabular}{|c|c|c|c|c|c|c|c|c|c|}
\hline $\mathrm{AmE}$ & \multicolumn{3}{|c|}{$1700 \mathrm{~s}$} & \multicolumn{3}{|c|}{$1800 \mathrm{~s}$} & \multicolumn{3}{|c|}{$1900 \mathrm{~s}$} \\
\hline wh-/that & \multicolumn{3}{|l|}{192} & \multicolumn{3}{|l|}{232} & \multicolumn{3}{|l|}{238} \\
\hline positives - false positives - ambiguous & 164 & 28 & 0 & 192 & 39 & 1 & 210 & 27 & 1 \\
\hline zero & \multicolumn{3}{|l|}{72} & \multicolumn{3}{|l|}{72} & \multicolumn{3}{|l|}{46} \\
\hline positives - false positives & 20 & \multicolumn{2}{|c|}{52} & 8 & \multicolumn{2}{|c|}{64} & 5 & \multicolumn{2}{|c|}{41} \\
\hline \multicolumn{10}{|l|}{$\mathrm{BrE}$} \\
\hline wh-/that & \multicolumn{3}{|l|}{301} & \multicolumn{3}{|l|}{305} & \multicolumn{3}{|l|}{176} \\
\hline positives - false positives - ambiguous & 260 & 42 & 0 & 252 & 53 & 0 & 144 & 31 & 1 \\
\hline zero & \multicolumn{3}{|l|}{132} & \multicolumn{3}{|l|}{91} & \multicolumn{3}{|l|}{48} \\
\hline positives - false positives & 26 & \multicolumn{2}{|c|}{106} & 22 & \multicolumn{2}{|c|}{69} & 0 & \multicolumn{2}{|c|}{48} \\
\hline \multicolumn{10}{|l|}{ both varieties } \\
\hline wh-/that & \multicolumn{3}{|l|}{493} & \multicolumn{3}{|l|}{537} & \multicolumn{3}{|l|}{414} \\
\hline positives - false positives - ambiguous & 424 & 70 & 0 & 444 & 92 & 1 & 354 & 58 & 2 \\
\hline zero & \multicolumn{3}{|l|}{204} & \multicolumn{3}{|l|}{163} & \multicolumn{3}{|l|}{94} \\
\hline positives - false positives & 46 & & 58 & 30 & & 133 & 5 & & 39 \\
\hline
\end{tabular}

